\title{
The Supernova Type Ia Rate Evolution with SNLS
}

\author{
James D. Neill*, M. Sullivan ${ }^{\dagger}$, D. Balam*, C. J. Pritchet*, D. A. Howell ${ }^{\dagger}$, \\ K. Perrett ${ }^{\dagger}$, P. Astier**, E. Aubourg ${ }^{\ddagger}$,, S. Basa ${ }^{\uparrow}$, R. G. Carlberg ${ }^{\dagger}$, \\ A. Conley ${ }^{\dagger}$, S. Fabbroll, D. Fouchez ${ }^{\dagger \dagger}$, J. Guy**, I. Hook ${ }^{* *}$, R. Pain** \\ N. Palanque-Delabrouille ${ }^{\S}$, N. Regnault**, J. Rich ${ }^{\S}$, R. Taillet ${ }^{* *, \S}$, \\ G. Aldering If, P. Antilogus**, V. Arsenijevic\|, C. Balland", S. Baumont**,

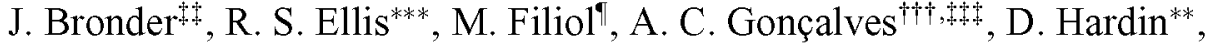 \\ M. Kowalski ${ }^{\text {†ा }}$, C. Lidman ${ }^{\S \S}$, V. Lusset ${ }^{\S}$, M. Mouchet ${ }^{\ddagger}$, A. Mourao" \\ S. Perlmutter ${ }^{\text {Tा }}$, P. Ripoche ${ }^{\dagger \dagger}$, D. Schlegel ${ }^{\text {Tा }}$ and C. Tao ${ }^{\dagger \dagger}$ \\ *University of Victoria, PO Box 3055 , Victoria, BC V8W 3P6, Canada \\ Iniversity of Toronto, 50 St. George Street, Toronto, ON M5S 3H4, Canada \\ ${ }^{* *}$ LPNHE, CNRS-IN2P3 and University of Paris VI \& VII, 75005 Paris, France \\ $¥ A P C, 11$ Pl. M. Berthelot, 75231 Paris Cedex 5, France

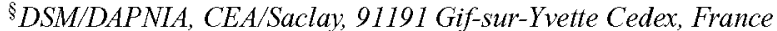 \\ "LAM CNRS, BP8, Traverse du Siphon, 13376 Marseille Cedex 12, France \\ $\|_{\text {CENTRA IST, Avenida Rovisco Pais, } 1049 \text { Lisbon, Portugal }}$ \\ ${ }^{\dagger}$ CPPM, CNRS-IN2P3 and Univ. Aix Marseille II, Case 907, 13288 Marseille Cedex 9, France \\ *University of Oxford Astrophysics, Denys Wilkinson Building, Keble Road, Oxford OXI 3RH, UK \\ $\$$ Université de Savoie, 73000 Chambéry, France \\ 1T LBNL, 1 Cyclotron Rd, Berkeley, CA 94720, USA \\ ${ }^{* * *}$ California Institute of Technology, E. California Blvd., Pasadena, CA 91125, USA \\ †† LUTH, UMR 8102, CNRS and Observatoire de Paris, F-92195 Meudon, France \\ \$ CAAUL, Observatório Astronómico de Lisboa, Tapada da Ajuda, 1349-018 Lisbon, Portugal \\ ${ }^{\$ \$}$ ESO, Alonzo de Cordova 3107, Vitacura, Casilla 19001, Santiago 19, Chile
}

\begin{abstract}
We present a progress report on a project to derive the evolution of the volumetric supernova Type Ia rate from the Supernova Legacy Survey. Our preliminary estimate of the rate evolution divides the sample from Neill et al. [1] into two redshift bins: $0.2<z<0.4$, and $0.4<$ $z<0.6$. We extend this by adding a bin from the sample analyzed in Sullivan et al. [2] in the range $0.6<z<0.75$ from the same time period. We compare the derived trend with previously published rates and a supernova Type Ia production model having two components: one component associated closely with star formation and an additional component associated with host galaxy mass. Our observed trend is consistent with this model, which predicts a rising SN Ia rate out to at least $z=2$.
\end{abstract}

Keywords: galaxies: evolution - galaxies: high redshift - supernovae: general

PACS: $97.60 . \mathrm{Bw}$

\section{INTRODUCTION}

The importance of Type Ia supernovae (SNe Ia) as cosmic distance tracers $[3,4]$ motivates the efforts to understand their progenitors. We need not only explain the physics that makes their explosions so useful, but we must also recognize and control the systematic effects that may result from the properties of their progenitors and their evolution.

CP924, The Multicolored Landscape of Compact Objects and their Explosive Origins - Cefalu 2006, edited by T. Di Salvo, L. A. Antonelli, G. L. Israel, L. Piersanti, A. Tomambe, L. Burderi, F. Fiore, G. Matt, and M. T. Menna O 2007 American Institute of Physics 978-0-7354-0434-2/07/\$23.00 
One method used to explore various progenitor models is to compare the evolution of star formation in the universe with the evolution of the observed SN Ia rate $[5,6,7$, $8,9,10,11,12]$. The challenge of this method is that it requires minimizing systematic effects that can produce spurious trends as a function of redshift. The redshift regions most important for constraining the progenitors, the highest and lowest redshifts, are also the most prone to systematic effects. At low redshifts cosmic variance and the difficulty of sampling large volumes and thus produce good statistics are severe challenges. At high redshifts, the faintness of the objects produce low signal-to-noise detections and less certain typing as well as higher host contamination since projected host offsets are smaller.

The Supernova Legacy Survey (SNLS) offers the opportunity to measure the SN Ia rate trend right in the sweet spot where the volume sampled per square degree is large and yet the objects are bright enough for high quality typing. This circumstance motivated the recently published study of Neill et al. [1] which produced an anchor point for rate evolution studies at $z \sim 0.5$. Our next step is to extend this effort by binning the original sample and adding a higher redshift bin to trace the rate evolution from $z=0.2$ to $z=0.75$. The homogeneous sample and high quality typing provided by SNLS can thus achieve a reduction in the systematics in this redshift range and provide a foundation for rate evolution studies at higher and lower redshifts.

Even though rigorous constraints of SN Ia progenitors await accurate low and high redshift rate measurements, we can still address a few questions that have arisen from other studies of SN Ia rates. We would like to see if we can reproduce the observed trends from previously published studies, in particular those that show a large jump in the SN Ia rate just beyond $z=0.5$ [12]. Of particular importance to future SN Ia surveys, such as JDEM and LSST, is the question of the rate evolution beyond $z=1$, which appears to decline dramatically [11]. This seems to contradict the observation in the local universe that the SN Ia rate is higher in star forming galaxies $[5,13,14,15]$ which implies that the SN Ia rate should increase at least out to $z \sim 2$. This correlation of SN Ia rate with host star formation rate has now been observed in the intermediate redshift universe using SNLS [2] which implies it is not a local effect. For this study we ask: is the observed trend in the SN Ia volumetric rate consistent with this correlation of rate with host properties?

\section{THE SUPERNOVA LEGACY SURVEY}

The SNLS was instigated with the goal of providing observational constraints on the pressure/density ratio of the universe, $w$. Its first-year results [16], in combination with other cosmological probes, provide one of the best observational constraints on $w$ [17]. It is designed as a rolling-search survey of four one-square-degree fields evenly spaced in RA. Each field is imaged with the Megacam imager on the Canada-France-Hawaii Telescope five times a month during its roughly six-month observing season in four wavelength bands: $g$ ' $r$ ' $i$ ' $z$ '. A deep $u^{*}$ image is also taken of each field to aid in measuring host galaxy properties. As each epoch is acquired it is scanned for variable objects 
TABLE 1. Spectroscopically confirmed SN Ia samples

\begin{tabular}{lrrr}
\hline Field & $0.2<z<0.4$ & $0.4<z<0.6$ & $0.6<z<0.75$ \\
\hline D1 & 3 & 13 & 9 \\
D2 & 5 & 10 & 12 \\
D3 & 6 & 10 & 12 \\
D4 & 3 & 8 & 9 \\
\hline ALL & 17 & 41 & 42 \\
\hline
\end{tabular}

which are recorded in a database ${ }^{1}$. SN-like variables are assessed for their SN Ia type likelihood [18] and all useful SN Ia candidates are promoted for followup spectroscopy with the VLT or the Gemini or Keck Telescopes. The availability of 8 and 10 meter class telescopes for spectroscopic followup provides a well defined completeness and a large spectroscopically confirmed sample of objects. The database of all variable objects provides a means to estimate spectroscopic completeness [1].

\section{RATE EVOLUTION WITH SNLS}

Neill et al. [1] used a Monte Carlo method to estimate the SNLS survey efficiency in the redshift range $0.2<z<0.6$. Updated host extinction models [19] improved the estimation of the systematic errors. The study of SN Ia rates as a function of host properties by Sullivan et al. [2] used the same sample and methods, but added an additional sample in the range $0.6<z<0.75$.

To estimate the rate evolution using the SNLS, we split the sample from [1] and [2] into three bins: $0.2<z<0.4,0.4<z<0.6$, and $0.6<z<0.75$. Separate Monte Carlo runs were performed for each sub-sample including experiments to determine the associated systematic errors in each redshift bin. Table 1 lists the spectroscopically confirmed sample for each field in each redshift bin. The preliminary results reported here will be improved upon by adding more recent SNe Ia from the survey to increase the sample size in each bin.

We applied the same methods used in [1] to derive the volumetric SN Ia rate in each bin, and associated systematic and statistical errors. We now compare the results to the two-component model of SN Ia production $[2,15,20]$.

\section{THE TWO-COMPONENT MODEL}

This model proposes a component associated with the host galaxy mass, to explain the non-zero SN Ia rate observed in early-type galaxies, and a component associated with the host galaxy star formation rate, to explain the correlation of SN Ia rate with host star formation rate $[2,15]$. The volumetric SN Ia rate evolution, $r_{V}(t)$, is thus expressed as

\footnotetext{
${ }^{1}$ see http://legacy.astro.utoronto.ca, and http://makiki.cfht.hawaii.edu: $872 /$ sne $/$
} 


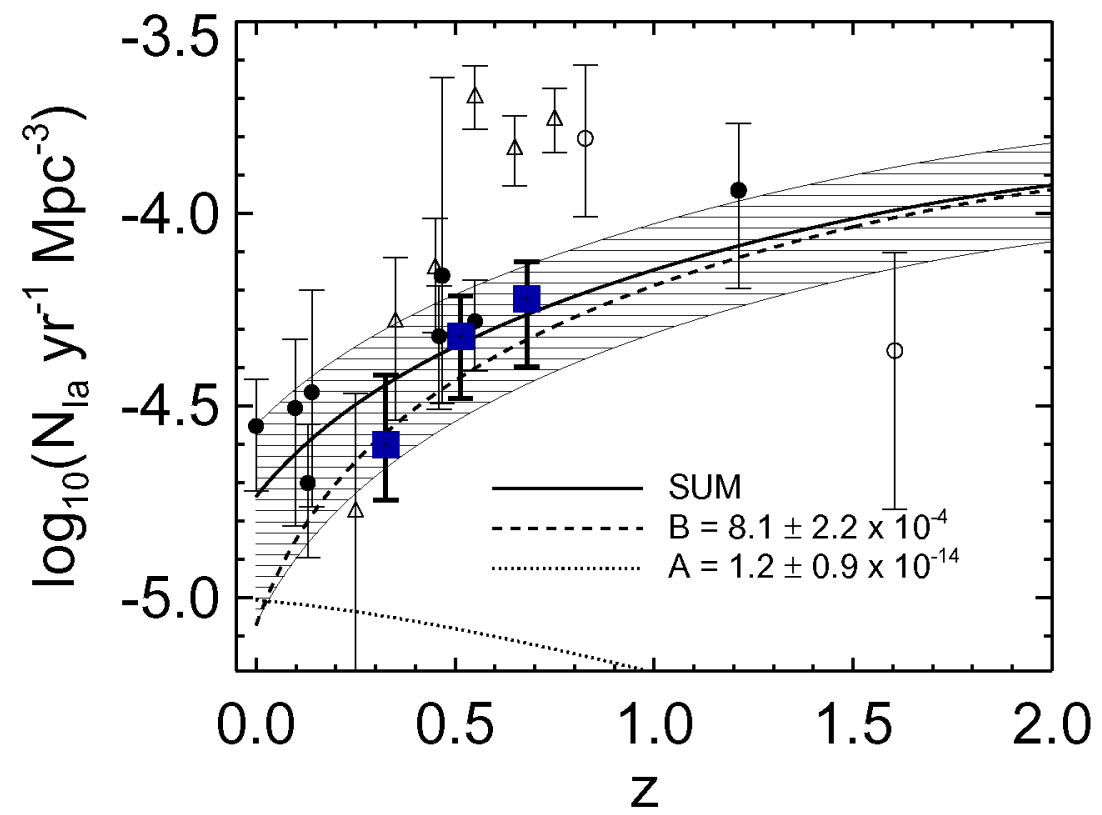

FIGURE 1. Observed SNLS SN Ia volumetric rate evolution. The filled squares are the SNLS rates in each of the three redshift bins. The filled circles are previously published SN Ia rates derived from samples primarily confirmed by spectroscopy from the following references (in redshift order): $[5,8,10$, $6,9,11,7,11]$. The open circles are the SN Ia rates from [11] with samples having only $50 \%$ spectroscopic confirmation. The open triangles are the rates from [12], whose samples are confirmed almost entirely with photometric methods. Error bars represent the systematic and statistical errors added in quadrature.

follows:

$$
r_{V}(t)=A M_{*}(t)+B \dot{M}_{*}(t),
$$

with $A$ in terms of SNe Ia per year per unit host stellar mass and $B$ in terms of SNe Ia per year per unit host star formation. The $B$ component produces SNe Ia on shorter $(\sim 1 \mathrm{Gyr})$ time-scales, while the $A$ component is responsible for the longer time-scale required to produce $\mathrm{SNe}$ Ia in galaxies dominated by older stellar populations.

Figure 1 shows a fit (solid line) of the SNLS rate evolution (filled squares) and various rates from the literature (solid circles) to the two-component model based on the star formation history (SFH) as parameterized by Hopkins and Beacom [21]. The resulting coefficients are annotated on the plot and the resulting evolution of each component is indicated by the dashed line ( $B$ component) and the dotted line ( $A$ component). The statistical uncertainty in the combined components is indicated by the hashed region.

We note that this model appears to be incompatible with two features in the observed rates. This fit is statistically inconsistent with the large jump in the rates seen at $z=0.5$ by Barris and Tonry [12]. It also predicts an increase in the SN Ia rate out to $z>2$, while the rates from Dahlen et al. [11] show a steady decline after a redshift of $z \sim 1$. The highest redshift observation $(z=1.6)$ is only marginally inconsistent with the model. 
The mass used in fitting the $A$ and $B$ components was derived by integrating the SFH from Hopkins and Beacom [21], and thus includes the mass of dead stars. When fitting host properties, the mass derived does not include dead stars. Our fitted values for $A$ and $B$ are consistent with published values $[1, \S 6.1 .1]$, but are marginally offset from host property values as expected from the differences in host masses. Our final results will include a mass correction for dead stars and thus produce $A$ and $B$ values more consistent with those derived from host properties.

\section{CONCLUSIONS}

By restricting our measurements of the SN Ia volumetric rate evolution to samples whose members are primarily identified with spectroscopy, we find a trend that appears compatible with the observed properties of SN Ia hosts. The most stringent test of this model awaits further measurements of the rate beyond $z=1$, but we must be careful. If the two-component model is correct, the higher redshift SNe Ia are more closely associated with star formation. This close association could introduce systematics from the dust and other features of high-redshift star formation that compound the difficulty of deriving accurate high-redshift SN Ia rates. This caution applies to the use of these high-redshift SNe Ia for cosmology as well.

\section{ACKNOWLEDGMENTS}

The authors wish to recognize and acknowledge the very significant cultural role and reverence that the summit of Mauna Kea has always had within the indigenous Hawaiian community. We are grateful for our opportunity to conduct observations on this mountain. We acknowledge invaluable assistance from the CFHT Queued Service Observations team, led by P. Martin (CFHT). Our research would not be possible without the assistance of the support staff at CFHT, especially J.-C. Cuillandre. The real-time pipelines for supernovae detection run on computers integrated in the CFHT computing system, and are very efficiently installed, maintained and monitored by K. Withington (CFHT). We also heavily rely on the real-time Elixir pipeline which is operated and monitored by J.-C. Cuillandre, E. Magnier and K. Withington. We are grateful to L. Simard (CADC) for setting up the image delivery system and his kind and efficient responses to our suggestions for improvements. The Canadian collaboration members acknowledge support from NSERC and CIAR; French collaboration members from CNRS/IN2P3, CNRS/INSU, PNC and CEA. This work was supported in part by the Director, Office of Science, Office of High Energy and Nuclear Physics, of the US Department of Energy. The France-Berkeley Fund provided additional collaboration support. We are indebted to A. Hopkins and J. Beacom for providing us with a draft of their work on SFH prior to its publication. The views expressed in this article are those of the author and do not reflect the official policy or position of the United States Air Force, Department of Defense, or the U.S. Government. 


\section{REFERENCES}

1. J. D. Neill, et al., $A J$ 132, 1126-1145(2006).

2. M. Sullivan, et al., ApJ 648, 868-883 (2006).

3. A. G. Riess, et al., $A J 116,1009-1038$ (1998).

4. S. Perlmutter, et al., ApJ 517, 565-586 (1999).

5. E. Cappellaro, R. Evans, and M. Turatto, $A \& A$ 351, 459-466 (1999).

6. D. Hardin, et al., $A \& A 362,419-425$ (2000).

7. R. Pain, et al., ApJ 577, 120-132(2002).

8. D. S. Madgwick, P. C. Hewett, D. J. Mortlock, and L. Wang, ApJL 599, L33-L36 (2003).

9. J. L. Tonry, et al., ApJ 594, 1-24 (2003).

10. G. Blanc, et al., $A \& A$ 423, 881-894 (2004).

11. T. Dahlen, et al., ApJ 613, 189-199 (2004).

12. B. J. Barris, and J. L. Tonry, ApJ 637, 427-438 (2006).

13. A. Oemler, and B. M. Tinsley, $A J$ 84, $985-992$ (1979).

14. S. van den Bergh, PASP 102, 1318-1320 (1990).

15. F. Mannucci, M. della Valle, N. Panagia, E. Cappellaro, G. Cresci, R. Maiolino, A. Petrosian, and M. Turatto, $A \& A 433,807-814$ (2005).

16. P. Astier, et al., $A \& A 447,31-48$ (2006).

17. D. N. Spergel, et al., $A p J$, submitted, astro-ph/0603449.

18. M. Sullivan, et al., $A J 131,960-972$ (2006).

19. M. Riello, and F. Patat, MNRAS 362, 671-680 (2005).

20. E. Scannapieco, and L. Bildsten, ApJL 629, L85-L88 (2005).

21. A. M. Hopkins, and J. F. Beacom, ApJ, submitted, astro-ph/0601463. 